\title{
Minimal $P$-symmetric period problem of first-order autonomous Hamiltonian Systems
}

\author{
Chungen Liu and Ben-Xing Zhou \\ School of Mathematics and LPMC, Nankai University, \\ Tianjin 300071, P. R. China
}

\begin{abstract}
Let $P \in S p(2 n)$ satisfying $P^{k}=I_{2 n}$, we consider the minimal $P$-symmetric period problem of the autonomous nonlinear Hamiltonian system

$$
\dot{x}(t)=J H^{\prime}(x(t)) .
$$

For some symplectic matrices $P$, we show that for any $\tau>0$ the above Hamiltonian system possesses a $k \tau$ periodic solution $x$ with $k \tau$ being its minimal $P$-symmetric period provided $H$ satisfies the Rabinowitz's conditions on the minimal period conjecture, together with that $H$ is convex and $H(P x)=H(x)$.
\end{abstract}

Key Words: Maslov $P$-index, Relative Morse index, Minimal $P$-symmetric period, Hamiltonian system

\section{Introduction and main result}

In this paper, we study the following first-order autonomous Hamiltonian system with $P$ boundary condition:

$$
\left\{\begin{array}{l}
\dot{x}=J H^{\prime}(x), x \in \mathbb{R}^{2 n} \\
x(\tau)=P x(0)
\end{array}\right.
$$

where $\tau>0, P \in S p(2 n)$, and $H \in C^{2}\left(\mathbb{R}^{2 n}, \mathbb{R}\right)$ is the Hamiltonian function satisfying $H(P x)=$ $H(x), \forall x \in \mathbb{R}^{2 n}$. $H^{\prime}(x)$ denote its gradient, $J=\left(\begin{array}{cc}0 & -I_{n} \\ I_{n} & 0\end{array}\right)$ is the standard symplectic matrix, $I_{n}$ is the identity matrix on $\mathbb{R}^{n}$. Without confusion, we shall omit the subindex of the identity matrix.

A solution $(\tau, x)$ of the problem (1.1) is called a $P$-solution of the Hamiltonian systems. The problem (1.1) has relation with the closed geodesics on Riemannian manifold (cf.[17]) and symmetric periodic solution or the quasi-periodic solution problem (cf.[18]). In addition, the

\footnotetext{
${ }^{1}$ Partially supported by the NSF of China (11471170, 10621101), 973 Program of MOST (2011CB808002) and SRFDP. Email: liucg@nankai.edu.cn(for Liu) and 1120130022@mail.nankai.edu.cn(for Zhou)
} 
first author C. Liu in [22] transformed some periodic boundary problem for nonlinear delay differential systems and some nonlinear delay Hamiltonian systems to $P$-boundary problems of Hamiltonian systems as above, we also refer [6, 14, 16, 19] and references therein for the background of $P$-boundary problems in $N$-body problems.

Let $P \in S p(2 n)$ and $k \in \mathbb{N}=\{0,1,2, \cdots\}$, we say $P$ satisfies $(P)_{k}$ condition, if $P^{k}=I_{2 n}$ and for each integer $m$ with $1 \leq m \leq k-1, P^{m} \neq I$. If $P$ satisfies $(P)_{k}$ condition, a $P$-solution $(\tau, x)$ can be extended as a $k \tau$-periodic solution $\left(k \tau, x^{k}\right)$. We say that a $T$-periodic solution $(T, x)$ of the Hamiltonian system in (1.1) is $P$-symmetric if $x\left(\frac{T}{k}\right)=P x(0) . T$ is the $P$-symmetric period of $x$. $T$ is called the minimal $P$-symmetric period of $x$ if $T=\min \left\{\lambda>0 \mid x\left(t+\frac{\lambda}{k}\right)=P x(t), \forall t \in \mathbb{R}\right\}$.

We assume the following conditions on $H$ in our arguments:

(H0) $H \in C^{1}\left(\mathbb{R}^{2 n}, \mathbb{R}\right) \forall x \in \mathbb{R}^{2 n}$;

(H1) $H \in C^{2}\left(\mathbb{R}^{2 n}, \mathbb{R}\right)$ with $H(P x)=H(x), \forall x \in \mathbb{R}^{2 n}$;

(H2) $H(x) \geq 0, \forall x \in \mathbb{R}^{2 n}$;

(H3) $H(x)=o\left(|x|^{2}\right)$ as $|x| \rightarrow 0$;

(H4) There are constants $\mu>2$ and $R_{0}>0$ such that

$$
0<\mu H(x) \leq\left(H^{\prime}(x), x\right), \quad \forall|x| \geq R_{0}
$$

(H5) $H^{\prime \prime}(x)>0, \forall x \in \mathbb{R}^{2 n}$;

In [35], Rabinowitz proved that the Hamiltonian system in (1.1) possesses a non-constant prescribed period solution provided $H$ satisfying $(\mathrm{H} 0)$ and $(\mathrm{H} 2)-(\mathrm{H} 4)$. Because a $\tau / k$-periodic function is also a $\tau$-periodic function, moreover, in [35] Rabinowitz proposed a conjecture: under the conditions (HO) and (H2)-(H4), for any $\tau>0$, the Hamiltonian system in (1.1) possesses a $\tau$-periodic with $\tau$ being its minimal period. Since then, there were many papers on this minimal period problem (cf. [5], 2], 1] , [10], [31], 32], [33], [9], etc.). In 1997, D. Dong and Y. Long 9] developed a new method on this prescribed minimal period solution problem and discovered the intrinsic relationship between the minimal period and the indices of a solution. Based upon the work of [9], G. Fei, Q. Qiu, T. Wang and others applied this method to various problems of Rabinowitz's conjecture (cf. [12], [13], [24], etc.). In fact, under conditions $H(0)=0$ and $H(x)>0, \forall x \in \mathbb{R}^{2 n} \backslash\{0\} ; \frac{H(x)}{|x|^{2}} \rightarrow+\infty$ as $x \rightarrow 0$ and $\frac{H(x)}{|x|^{2}} \rightarrow 0$ as $x \rightarrow+\infty$, F. Clarke and I. Ekeland proved a result on the corresponding minimal period problem for some given $T$ in [5]; I. Ekeland and H. Hofer gave a criterion for the conjecture in [10] which is unfortunately not easy to check.

For $P$-boundary problem, S. Tang [28] and the first author of this paper proved that for any $0<\tau<\frac{\pi}{\max _{t \in[0, \tau]}\left\|\dot{\gamma}_{P}(t) \gamma_{P}(t)^{-1}\right\|}$, there exists a nonconstant $P$-solution with its minimal $P$ symmetric period $k \tau$ or $\frac{k \tau}{k+1}$ via the iteration theory of Maslov $P$-index. In [23], the first author 
of this paper improved the result that for every $\tau>0$, there exists a nonconstant $P$-solution with its minimal $P$-symmetric period $k \tau$ or $\frac{k \tau}{k+1}$.

For $n \in \mathbb{N}, k>0$, denote by

$$
\begin{gathered}
S p(2 n) \equiv S p(2 n, \mathbb{R})=\left\{M \in \mathcal{L}\left(\mathbb{R}^{2 n}\right) \mid M^{T} J M=J\right\}, \\
\mathcal{P}_{\tau}(2 n) \equiv\{\gamma \in C([0, \tau], S p(2 n)) \mid \gamma(0)=I\}, \\
S p(2 n)_{k} \equiv\left\{P \in S p(2 n) \mid P \text { satisfies }(P)_{k} \text { condition }\right\}, \\
\Omega(M) \equiv\left\{N \in S p(2 n) \mid \sigma(\mathrm{N}) \cap \mathbf{U}=\sigma(\mathrm{M}) \cap \mathbf{U} \text { and } \nu_{\lambda}(\mathrm{N})=\nu_{\lambda}(\mathrm{M}), \forall \lambda \in \sigma(\mathrm{M}) \cap \mathbf{U}\right\} .
\end{gathered}
$$

Denote by $\Omega^{0}(M)$ the path connected component of $\Omega(M)$ which contains $M$.

Lemma 1.1. If $P \in S p(2 n)_{k}$, then there exists a matrix $I_{2 p} \diamond R\left(\frac{2 \pi}{k}\right)^{\diamond j_{1}} \diamond \cdots \diamond R\left(\frac{2 r \pi}{k}\right)^{\diamond j_{r}} \in \Omega^{0}\left(P^{-1}\right)$, with $p+\sum_{m=1}^{r} j_{m}=n$.

Proof. For $P \in S p(2 n)_{k}$, we have $\sigma\left(P^{-1}\right)=\sigma(P) \subseteq\left\{1, e^{\frac{2 \pi \sqrt{-1}}{k}}, e^{\frac{4 \pi \sqrt{-1}}{k}}, \cdots, e^{\frac{2(k-1) \pi \sqrt{-1}}{k}}\right\} \subseteq \mathbf{U}$. By the Theorem 1.8.10 in [29], there exists $M_{1}\left(\omega_{1}\right) \diamond M_{2}\left(\omega_{2}\right) \diamond \cdots \diamond M_{s}\left(\omega_{s}\right) \in \Omega^{0}\left(P^{-1}\right)$ where $M_{i}\left(\omega_{i}\right)$ is a basic normal form of some eigenvalue of $P^{-1}, 1 \leq i \leq s$. And the following are the basic normal forms for eigenvalues in $\mathbf{U}$.

Case 1. $N_{1}(\lambda, b)=\left(\begin{array}{cc}\lambda & b \\ 0 & \lambda\end{array}\right), \lambda= \pm 1, b= \pm 1,0$.

Since $P \in S p(2 n)_{k}$, we have $b=0$ and $\lambda \in\{-1,1\} \cap \sigma\left(P^{-1}\right)$.

Case 2. $R(\theta)=\left(\begin{array}{cc}\cos \theta & -\sin \theta \\ \sin \theta & \cos \theta\end{array}\right), \theta \in(0, \pi) \cup(\pi, 2 \pi)$.

Since $P \in S p(2 n)_{k}$, we have $\theta \in\left\{\frac{2 \pi}{k}, \frac{4 \pi}{k}, \cdots \frac{2(k-1) \pi}{k}\right\}$.

Case 3. $N_{2}(\omega, b)=\left(\begin{array}{cc}R(\theta) & b \\ 0 & R(\theta)\end{array}\right), \theta \in(0, \pi) \cup(\pi, 2 \pi), b=\left(\begin{array}{cc}b_{1} & b_{2} \\ b_{3} & b_{4}\end{array}\right), b_{i} \in \mathbb{R}, b_{2} \neq b_{3}$.

From direct computation, it is easy to check that the matrix $T=\left(\begin{array}{cc}\frac{1}{\sqrt{2}} & \frac{\sqrt{-1}}{\sqrt{2}} \\ \frac{\sqrt{-1}}{\sqrt{2}} & \frac{1}{\sqrt{2}}\end{array}\right)$ satisfies that $\operatorname{TR}(\theta) T^{-1}=\left(\begin{array}{cc}e^{\sqrt{-1} \theta} & 0 \\ 0 & e^{-\sqrt{-1} \theta}\end{array}\right)$. Then $\left(\begin{array}{cc}T & 0 \\ 0 & T\end{array}\right) N_{2}(\omega, b)\left(\begin{array}{cc}T^{-1} & 0 \\ 0 & T^{-1}\end{array}\right)=$ $\left(\begin{array}{cc}T R(\theta) T^{-1} & T b T^{-1} \\ 0 & T R(\theta) T^{-1}\end{array}\right)$, where

$$
T b T^{-1}=\left(\begin{array}{ll}
\frac{1}{2}\left(b_{1}+b_{4}\right)-\frac{\sqrt{-1}}{2}\left(b_{2}-b_{3}\right) & \frac{1}{2}\left(b_{2}+b_{3}\right)-\frac{\sqrt{-1}}{2}\left(b_{1}-b_{4}\right) \\
\frac{1}{2}\left(b_{2}+b_{3}\right)+\frac{\sqrt{-1}}{2}\left(b_{1}-b_{4}\right) & \frac{1}{2}\left(b_{1}+b_{4}\right)+\frac{\sqrt{-1}}{2}\left(b_{2}-b_{3}\right)
\end{array}\right) .
$$

Denoted by

$$
\left(\begin{array}{cc}
T R(i \theta) T^{-1} & X(i) \\
0 & T R(k \theta) T^{-1}
\end{array}\right)=\left(\begin{array}{cc}
T R(\theta) T^{-1} & T b T^{-1} \\
0 & T R(\theta) T^{-1}
\end{array}\right)^{i}, i \in \mathbb{N}
$$


where $X(i)=\left(\begin{array}{ll}x_{1}(i) & x_{2}(i) \\ x_{3}(i) & x_{4}(i)\end{array}\right)$ and $X(1)=T b T^{-1}=\left(\begin{array}{ll}x_{1}(1) & x_{2}(1) \\ x_{3}(1) & x_{4}(1)\end{array}\right)$. By direct computation, we have

$$
\begin{aligned}
& x_{1}(k)=k e^{\sqrt{-1}(k-1) \theta} x_{1}(1), \\
& x_{4}(k)=k e^{-\sqrt{-1}(k-1) \theta} x_{4}(1) .
\end{aligned}
$$

Thus, from $P^{k}=I$ we have $X(k)=0$, so $x_{1}(1)=x_{4}(1)=0$, i.e.

$$
\begin{aligned}
& \frac{1}{2}\left(b_{1}+b_{4}\right)-\frac{\sqrt{-1}}{2}\left(b_{2}-b_{3}\right)=0, \\
& \frac{1}{2}\left(b_{1}+b_{4}\right)+\frac{\sqrt{-1}}{2}\left(b_{2}-b_{3}\right)=0 .
\end{aligned}
$$

Then we have $b_{2}=b_{3}$, which is contradict to the definition of the basic normal form $N_{2}(\omega, b)$.

Therefore, from (Case1)-(Case3), we get $M_{i}\left(\omega_{i}\right)=R\left(\theta_{i}\right)$ where $\theta_{i} \in\left\{0, \frac{2 \pi}{k}, \frac{4 \pi}{k}, \cdots \frac{2(k-1) \pi}{k}\right\}$, $1 \leq i \leq s$. And the lemma is proved.

For the notations in Lemma 1.1, we define

$$
S p(2 n)_{k}\left(r, p ; j_{1}, j_{2}, \cdots, j_{r}\right) \equiv\left\{P \in S p(2 n)_{k} \mid k-2 \sum_{m=1}^{r} m \cdot j_{m}>1, r<\frac{k}{2}\right\} .
$$

Now we state the main result of this paper.

Theorem 1.1. Suppose $P \in S p(2 n)_{k}\left(r, p ; j_{1}, j_{2}, \cdots, j_{r}\right)$, and the Hamiltonian function $H$ satisfies (H1)-(H5), then for every $\tau>0$, the system (1.1) possesses a non-constant P-solution $(\tau, x)$ such that the minimal P-symmetric period of the extended $k \tau$-periodic solution $\left(k \tau, x^{k}\right)$ is $k \tau$.

In order to prove the above result, we need to obtain the relationship between the Maslov $P$-index and Morse index. Thus we organize this paper as follows, in Section 2, we recall the definition and properties of the Maslov $P$-index theory, and we also list out the relationship between the Morse index and the Maslov P-index (see [27], 28], 23], 11] and [12]). In Section 3 , we first study the iteration formila of Maslov index of paths $\xi \in \mathcal{P}_{\tau}(2 n)$ such that $\xi(\tau)=P^{-1}$ in detail, then we will give the complete proof of the main result.

\section{Preliminaries}

In this section, we give a brief introduction to the Maslov $P$-index and its iteration properties, and then give the relationship between Maslov $P$-index and the relative Morse index which is studied by the first author of this paper in [23].

Maslov $P$-index was first studied in [7] and [21] independently for any symplectic matrix $P$ with different treatment. The first author and S. Tang in [27, 28] defined the Maslov $(P, \omega)$-index $\left(i_{\omega}^{P}(\gamma), \nu_{\omega}^{P}(\gamma)\right)$ for any symplectic path $\gamma \in \mathcal{P}_{\tau}(2 n)$. And then the first author of this paper used relative index theory to develop Maslov $P$-index in [23] which is consistent with the definition 
in [27, 28]. When the symplectic matrix $P=\operatorname{diag}\left\{-I_{n-\kappa}, I_{\kappa},-I_{n-\kappa}, I_{\kappa}\right\}, 0 \leq \kappa \in \mathbb{N} \leq n$, the $(P, \omega)$-index theory and its iteration theory were studied in [8] and then be successfully used to study the multiplicity of closed characteristics on partially symmetric convex compact hypersurfaces in $\mathbb{R}^{2 n}$. Here we use the notions and results in [21, 27, 28].

For $\omega \in \mathbf{U}$, then the Maslov $(P, \omega)$-index of a symplectic path $\gamma \in \mathcal{P}_{\tau}(2 n)$ is defined as a pair of integers(cf.[27])

$$
\left(i_{\omega}^{P}(\gamma), \nu_{\omega}^{P}(\gamma)\right) \in \mathbb{Z} \times\{0,1, \cdots, 2 n\}
$$

where the index part

$$
i_{\omega}^{P}(\gamma)=i_{\omega}\left(P^{-1} \gamma * \xi\right)-i_{\omega}(\xi)
$$

$\xi \in \mathcal{P}_{\tau}(2 n)$ such that $\xi(\tau)=P^{-1}$ and the nullity

$$
\nu_{\omega}^{P}(\gamma)=\operatorname{dim} \operatorname{ker}(\gamma(\tau)-\omega P)
$$

Suppose $B(t) \in C\left(\mathbb{R}, \mathcal{L}_{s}\left(\mathbb{R}^{2 n}\right)\right)$, if $\gamma \in \mathcal{P}_{\tau}(2 n)$ is the fundamental solution of the linear Hamiltonian systems

$$
\dot{y}(t)=J B(t) y, \quad y \in \mathbb{R}^{2 n},
$$

we also call $\left(i_{\omega}^{P}(\gamma), \nu_{\omega}^{P}(\gamma)\right)$ the Maslov $(P, \omega)$-index of $B(t)$, denoting $\left(i_{\omega}^{P}(B), \nu_{\omega}^{P}(B)\right)=\left(i_{\omega}^{P}(\gamma), \nu_{\omega}^{P}(\gamma)\right)$, just as in [21, 27, 28]. If $x$ is a $P$-solution of (1.1), then the Maslov $(P, \omega)$-index of the solution $x$ is defined to be the Maslov $(P, \omega)$-index of $B(t)=H^{\prime \prime}(x(t))$ and denoted by $\left(i_{\omega}^{P}(x), \nu_{\omega}^{P}(x)\right)$. When $\omega=1$, we omit the subindex, denoted by $\left(i^{P}(\gamma), \nu^{P}(\gamma)\right)$ or $\left(i^{P}(B), \nu^{P}(B)\right)$ for simplicity.

For $m \in \mathbb{N}$, we extend the definition of $x(t)$ which is the solution of $(1.1)$ to $[0,+\infty)$ by

$$
x(t)=P^{j} x(t-j \tau), \quad \forall j \tau \leq t \leq(j+1) \tau, j \in \mathbb{N}
$$

and define the $m$-th iteration $x^{m}$ of $x$ by

$$
x^{m}=\left.x\right|_{[0, m \tau]} .
$$

If $P$ satisfies $(P)_{k}$ condition, then $x^{k}$ becomes an $k \tau$-periodic solution of the Hamiltonian system in (1.1). We know that the fundamental solution $\gamma_{x} \in \mathcal{P}_{\tau}(2 n)$ carries significant information about $x$. For any $\gamma \in \mathcal{P}_{\tau}(2 n)$, S. Tang and the first author of this paper have defined the corresponding $m$-th iteration path $\gamma^{m}:[0, m \tau] \rightarrow S p(2 n)$ of $\gamma$ in [27] by

$$
\gamma^{m}(t)= \begin{cases}\gamma(t), & t \in[0, \tau], \\ P \gamma(t-\tau) P^{-1} \gamma(\tau), & t \in[\tau, 2 \tau], \\ P^{2} \gamma(t-2 \tau)\left(P^{-1} \gamma(\tau)\right)^{2}, & t \in[2 \tau, 3 \tau], \\ P^{3} \gamma(t-3 \tau)\left(P^{-1} \gamma(\tau)\right)^{3}, & t \in[3 \tau, 4 \tau], \\ \cdots \cdots & \\ P^{m-1} \gamma(t-(m-1) \tau)\left(P^{-1} \gamma(\tau)\right)^{m-1}, & t \in[(m-1) \tau, m \tau] .\end{cases}
$$

If the matrix function $B(t)$ in the linear Hamiltonian system (2.3) satisfies $P^{T} B(t+\tau) P=B(t)$, the iteration of its fundamental solution $\gamma$ is defined in the same way. 
Corresponding we set

$$
i_{\omega}^{P^{m}}(\gamma, m)=i_{\omega}^{P^{m}}\left(\gamma^{m}\right), \quad \nu_{\omega}^{P^{m}}(\gamma, m)=\nu_{\omega}^{P^{m}}\left(\gamma^{m}\right)
$$

If the subindex $\omega=1$, we simply write $\left(i^{P^{m}}(\gamma, m), \nu^{P^{m}}(\gamma, m)\right)$, and omit the subindex 1 when there is no confusion. In the sequel, we use the notions $(i(\gamma), \nu(\gamma))$ and $(i(\gamma, m), \nu(\gamma, m))$ to denote the Maslov-type index and the iterated index of symplectic path $\gamma$ with the periodic boundary condition which were introduced by Y. Long and his collaborators (cf. [25], [26], [29], [34], etc.).

In [27, 28], S. Tang and the first author of this paper obtained the important Bott-type formula and iteration inequalities for Malsov $(P, \omega)$-index as follows.

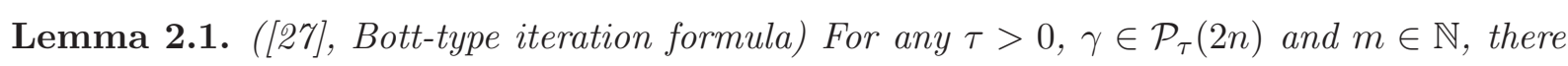
hold

$$
i_{\omega_{0}}^{P^{m}}(\gamma, m)=\sum_{\omega^{m}=\omega_{0}} i_{\omega}^{P}(\gamma), \quad \nu_{\omega_{0}}^{P^{m}}(\gamma, m)=\sum_{\omega^{m}=\omega_{0}} \nu_{\omega}^{P}(\gamma)
$$

where $P \in S p(2 n)$ and $\omega_{0} \in \mathbf{U}$.

Lemma 2.2. ([28]) For any path $\gamma \in \mathcal{P}_{\tau}(2 n), P \in S p(2 n)$ and $\omega \in \mathbf{U} \backslash\{1\}$, it always holds that

$$
i^{P}(\gamma, 1)+\nu^{P}(\gamma, 1)-n+i_{1}(\xi)-i_{\omega}(\xi) \leq i_{\omega}^{P}(\gamma) \leq i^{P}(\gamma, 1)+n-\nu_{\omega}^{P}(\gamma)+i_{1}(\xi)-i_{\omega}(\xi) .
$$

Lemma 2.3. ([28], iteration inequality) For any path $\gamma \in \mathcal{P}_{\tau}(2 n), P \in S p(2 n)$ and $m \in \mathbf{N}$,

$$
\begin{aligned}
& m\left(i^{P}(\gamma, 1)+\nu^{P}(\gamma, 1)-n\right)+n-\nu^{P}(\gamma, 1)+m i_{1}(\xi)-i(\xi, m) \\
& \leq i^{P^{m}}(\gamma, m) \\
& \leq m\left(i^{P}(\gamma, 1)+n\right)-n-\left(\nu^{P^{m}}(\gamma, m)-\nu^{P}(\gamma, 1)\right)+m i_{1}(\xi)-i(\xi, m) .
\end{aligned}
$$

Let $e(M)$ be the elliptic height of symplectic matrix $M$ just as the same in [29], the following lemma is important for the proof of Theorem 1.1 .

Lemma 2.4 ([28]). For any path $\gamma \in \mathcal{P}_{\tau}(2 n), P \in S p(2 n)$, set $M=\gamma(\tau)$ and extend $\gamma$ to $[0, \infty)$ by 2.4). Then for any $m \in \mathrm{N}$ we have

$$
\begin{aligned}
& \nu^{P^{m}}(\gamma, m)-\nu(\xi, 1)+\nu(\xi, m+1)-\frac{e\left(P^{-1} M\right)}{2}-\frac{e\left(P^{-1}\right)}{2} \\
& \leq i^{P^{(m+1)}}(\gamma, m+1)-i^{P^{m}}(\gamma, m)-i^{P}(\gamma, 1) \\
& \leq \nu^{P}(\gamma, 1)-\nu^{P^{(m+1)}}(\gamma, m+1)-\nu(\xi, m)+\frac{e\left(P^{-1} M\right)}{2}+\frac{e\left(P^{-1}\right)}{2} .
\end{aligned}
$$

Let $S_{k \tau}=\mathbb{R} /(k \tau \mathbb{Z}), W_{P}=\left\{z \in W^{1 / 2,2}\left(S_{k \tau}, \mathbb{R}^{2 n}\right) \mid z(t+\tau)=P z(t)\right\}$ be a closed subspace of $W^{1 / 2,2}\left(S_{k \tau}, \mathbb{R}^{2 n}\right)$. It is also a Hilbert space with norm $\|\cdot\|$ and inner product $\langle\cdot, \cdot\rangle$ as in $W^{1 / 2,2}\left(S_{k \tau}, \mathbb{R}^{2 n}\right)$. We denote by $\|\cdot\|_{s}$ the $L^{s}$-norm for $s \geq 1$. By the well-known Sobolev embedding theorem, we have the following embedding property: for any $s \in[1,+\infty)$, there is a constant $\alpha_{s}>0$ such that

$$
\|z\|_{s} \leq \alpha_{s}\|z\|, \quad \forall z \in W_{P}
$$


Let $\mathcal{L}_{s}\left(W_{P}\right)$ and $\mathcal{L}_{c}\left(W_{P}\right)$ denote the space of the bounded self-adjoint linear operator and compact linear operator on $W_{P}$. We define two operators $A, B \in \mathcal{L}_{s}\left(W_{P}\right)$ by the following bilinear forms:

$$
\langle A x, y\rangle=\int_{0}^{\tau}(-J \dot{x}(t), y(t)) d t, \quad\langle B x, y\rangle=\int_{0}^{\tau}(B(t) x(t), y(t)) d t .
$$

Suppose that $\cdots \leq \lambda_{-j} \leq \cdots \leq \lambda_{-1}<0<\lambda_{1} \leq \cdots \leq \lambda_{j} \leq \cdots$ are all nonzero eigenvalues of $A$ (count with multiplicity), correspondingly, $e_{j}$ is the eigenvector of $\lambda_{j}$ satisfying $\left\langle e_{j}, e_{i}\right\rangle=\delta_{j i}$. We denote the kernel of $A$ by $W_{P}^{0}$ which is exactly the $\operatorname{space}_{\operatorname{ker}_{\mathbb{R}}}(P-I)$. For $m \in \mathbb{N}$, define the finite dimensional subspace of $W_{P}$ by

$$
W_{P}^{m}=W_{m}^{-} \oplus W_{P}^{0} \oplus W_{m}^{+}
$$

with $W_{m}^{-}=\left\{z \in W_{P} \mid z(t)=\sum_{j=1}^{m} a_{-j} e_{-j}(t), a_{-j} \in \mathbb{R}\right\}$ and $W_{m}^{+}=\left\{z \in W_{P} \mid z(t)=\sum_{j=1}^{m} a_{j} e_{j}(t)\right.$, $\left.a_{j} \in \mathbb{R}\right\}$. Suppose $P_{m}$ is the orthogonal projections $P_{m}: W_{P} \rightarrow W_{P}^{m}$ for $m \in \mathbb{N} \cup\{0\}$. Then $\left\{P_{m} \mid m=0,1,2, \cdots\right\}$ is the Galerkin approximation sequence respect to $A$.

For a self-adjoint operator $T$, we denote by $M^{*}(T)$ the eigenspaces of $T$ with eigenvalues belonging to $(0,+\infty),\{0\}$ and $(-\infty, 0)$ with $*=+, 0$ and $*=-$, respectively. And the dimension of eigenspaces $M^{*}(T)$ is denoted by $m^{*}(T)=\operatorname{dim} M^{*}(T)$. Similarly, we denote by $M_{d}^{*}(T)$ the eigenspaces of $T$ with eigenvalues belonging to $(d,+\infty),(-d, d)$ and $(-\infty,-d)$ with $*=+, 0$ and $*=-$, respectively. we denote $m_{d}^{*}(T)=\operatorname{dim} M_{d}^{*}(T)$. For any adjoint operator $L$, we denote $L^{\sharp}=\left(\left.L\right|_{I m L}\right)^{-1}$.

The following theorem gives the relationship between the Maslov $P$-index and Morse index for any $P \in S p(2 n)$.

Theorem 2.1. ([23], Lemma 3.2 and Theorem 4.6) For $P \in S p(2 n)$, suppose that $B(t) \in$ $\mathbb{C}\left(\mathbb{R}, \mathcal{L}_{s}\left(\mathbb{R}^{2 n}\right)\right)$ and $P^{T} B(t+\tau) P=B(t)$ with the Maslov $P$-index $\left(i^{P}(B), \nu^{P}(B)\right)$. For any constant $0<d \leq \frac{1}{4}\left\|(A-B)^{\sharp}\right\|^{-1}$, there exists an $m_{0}>0$ such that for $m \geq m_{0}$, there holds

$$
\begin{aligned}
& m_{d}^{-}\left(P_{m}(A-B) P_{m}\right)=m+i^{P}(B), \\
& m_{d}^{0}\left(P_{m}(A-B) P_{m}\right)=\nu^{P}(B), \\
& m_{d}^{+}\left(P_{m}(A-B) P_{m}\right)=m+\operatorname{dim} \operatorname{ker}_{\mathbb{R}}(P-I)-i^{P}(B)-\nu^{P}(B),
\end{aligned}
$$

where $B$ is the operator defined by $B(t)$.

For the operators $A$ and $B$ defined in (2.10), there is another description of the Maslov $P$-index as follows.

Lemma 2.5 ([23] $)$. For any two operators $B_{1}, B_{2} \in C\left(\mathbb{R}, \mathcal{L}_{s}(2 n)\right)$ with $B_{i}(t+\tau)=\left(P^{-1}\right)^{T} B_{i}(t) P^{-1}, i=$ 1,2 and $B_{1}<B_{2}$, there holds

$$
i^{P}\left(B_{2}\right)-i^{P}\left(B_{1}\right)=\sum_{s \in[0,1)} \nu^{P}\left((1-s) B_{1}+s B_{2}\right) .
$$

Remark 2.1. Suppose that $B>0$, we have

$$
i^{P}(B)=\sum_{s \in[0,1)} \nu^{P}(s B)
$$




\section{The proof of Theorem 1.1}

In [23], the following result was proved.

Theorem 3.1. ([23]) Suppose $P \in S p(2 n)_{k}$, and the Hamiltonian function $H$ satisfies (H1)(H4), then for every $\tau>0$, the system (1.1) possesses a nonconstant P-solution $(\tau, x)$ satisfying

$$
\operatorname{dim} \operatorname{ker}_{\mathbb{R}}(\mathrm{P}-\mathrm{I})+2-\nu^{\mathrm{P}}(x) \leq i^{P}(x) \leq \operatorname{dim} \operatorname{ker}_{\mathbb{R}}(\mathrm{P}-\mathrm{I})+1 .
$$

Before the proof of Theorem [1.1, we need to get the information about the iteration Maslov index for paths connecting $I$ and $P^{-1}$. Firstly, from Lemma 1.1 we recall that for any $P \in$ $S p(2 n)_{k}$, there exists $\left(p, j_{1}, j_{2}, \cdots, j_{r}\right) \in \mathbb{N}^{r+1}$ such that $p+\sum_{m=1}^{r} j_{m}=n$ and $I_{2 p} \diamond R\left(\frac{2 \pi}{k}\right)^{\diamond j_{1}} \diamond$ $\cdots \diamond R\left(\frac{2 r \pi}{k}\right)^{\diamond j_{r}} \in \Omega^{0}\left(P^{-1}\right)$. We remind that here $\mathbb{N}=\{0,1, \cdots$,$\} .$

Lemma 3.1. For $P \in S p(2 n)_{k}\left(r, p ; j_{1}, j_{2}, \cdots, j_{r}\right)$ and $\xi \in \mathcal{P}_{\tau}(2 n)$ with $\xi(\tau)=P^{-1}$, there holds

$$
(k+1) i(\xi)-i(\xi, k+1)=\sum_{m=1}^{r}(k-2 m) j_{m}-k p .
$$

Proof. By Theorem 9.3.1 in [29](also [34]), we have

$$
\begin{aligned}
i(\xi, k+1)= & (k+1)\left(i(\xi)+S_{P^{-1}}^{+}(1)-C\left(P^{-1}\right)\right) \\
& +2 \sum_{\theta \in(0,2 \pi)} E\left(\frac{(k+1) \theta}{2 \pi}\right) S_{P^{-1}}^{-}\left(e^{\sqrt{-1} \theta}\right)-\left(S_{P^{-1}}^{+}(1)+C\left(P^{-1}\right)\right) \\
= & (k+1) i(\xi)+k S_{P^{-1}}^{+}(1)-(k+2) C\left(P^{-1}\right)+2 \sum_{\theta \in(0,2 \pi)} E\left(\frac{(k+1) \theta}{2 \pi}\right) S_{P^{-1}}^{-}\left(e^{\sqrt{-1} \theta}\right),
\end{aligned}
$$

where $S_{M}^{ \pm}(\omega)$ denote the splitting number of $M \in S p(2 n)$ at $\omega \in \mathbf{U}, C(M)=\sum_{\theta \in(0,2 \pi)} S_{M}^{-}\left(e^{\sqrt{-1} \theta}\right)$ and $E(a)=\min \{m \in \mathbb{Z} \mid m \geq a\}$. One can see these notions in Chapter 9 of [29].

For $P \in S p(2 n)_{k}\left(r, p ; j_{1}, j_{2}, \cdots, j_{r}\right), I_{2 p} \diamond R\left(\frac{2 \pi}{k}\right)^{\diamond j_{1}} \diamond \cdots \diamond R\left(\frac{2 r \pi}{k}\right)^{\diamond j_{r}} \in \Omega^{0}\left(P^{-1}\right)$ with $2 r<k$. By direct computation, for $\theta \in(0, \pi) \cup(\pi, 2 \pi)$, we have

$$
\begin{gathered}
S_{P^{-1}}^{+}(1)=p ; \\
C\left(P^{-1}\right)=\sum_{m=1}^{r} j_{m} ; \\
S_{P^{-1}}^{-}\left(e^{\sqrt{-1} \theta}\right)= \begin{cases}j_{m}, & \text { if } \theta=\frac{2 m \pi}{k}, 1 \leq m \leq r, e^{\frac{2 m \pi \sqrt{-1}}{k}} \in \sigma\left(P^{-1}\right) ; \\
0, & \text { otherwise. }\end{cases}
\end{gathered}
$$

So

$$
\begin{aligned}
\sum_{\theta \in(0,2 \pi)} E\left(\frac{(k+1) \theta}{2 \pi}\right) S_{P^{-1}}^{-}\left(e^{\sqrt{-1} \theta}\right) & =E\left(\frac{k+1}{k}\right) j_{1}+E\left(\frac{2(k+1)}{k}\right) j_{2}+\cdots+E\left(\frac{r(k+1)}{k}\right) j_{r} \\
& =2 j_{1}+3 j_{2}+\cdots+(r+1) j_{r} .
\end{aligned}
$$


Then

$$
\begin{aligned}
i(\xi, k+1) & =(k+1) i(\xi)+k p-(k+2)\left(j_{1}+j_{2}+\cdots+j_{r}\right)+2\left(2 j_{1}+3 j_{2}+\cdots+(r+1) j_{r}\right) \\
& =(k+1) i(\xi)+k p-(k-2) j_{1}-(k-4) j_{2}-\cdots-(k-2 r) j_{r},
\end{aligned}
$$

thus $(k+1) i(\xi)-i(\xi, k+1)=\sum_{m=1}^{r}(k-2 m) j_{m}-k p$.

Now we are ready to prove Theorem 1.1

\section{Proof of Theorem 1.1.}

Suppose that $\left(k \tau, x^{k}\right)$ is the $k \tau$-periodic solution extended by $P$-solution $(\tau, x)$ in Theorem 3.1. If $k \tau$ is not the minimal $P$-symmetric period of $\left(k \tau, x^{k}\right)$, i.e., $\tau>\min \{\lambda>0 \mid x(t+\lambda)=$ $P x(t), \forall t \in \mathbb{R}\}$, then there exists some $l \in \mathbb{N}$ such that

$$
T \equiv \frac{\tau}{l}=\min \{\lambda>0 \mid x(t+\lambda)=P x(t), \forall t \in \mathbb{R}\} .
$$

Thus $x(\tau-T)=x(0)$, both $(l-1) T$ and $k T$ are the period of $x$. Since $k T$ is the minimal $P$-symmetric period, we obtain $k T \leq(l-1) T$ and then $k \leq l-1$.

Note that $\left.x\right|_{[0, k T]}$ is the $k$-th iteration of $\left.x\right|_{[0, T]}$. Suppose $\gamma \in \mathcal{P}_{T}(2 n)$ is the fundamental solution of the following linear Hamiltonian system

$$
\dot{z}(t)=J B(t) z(t)
$$

with $B(t)=H^{\prime \prime}\left(\left.x\right|_{[0, T]}(t)\right)$. Suppose $\xi$ be any symplectic path in $\mathcal{P}_{T}(2 n)$ such that $\xi(T)=P^{-1}$, since $P^{k}=I$, then

$$
\nu(\xi, 1)=\nu(\xi, k+1)=\nu(\xi, l)
$$

All eigenvalues of $P$ and $P^{-1}$ are on the unit circle, then the elliptic height

$$
e\left(P^{-1}\right)=e(P)=2 n \text {. }
$$

Since the system (1.1) is autonomous, we have

$$
\nu_{1}\left(\left.x\right|_{[0, k T]}\right) \geq 1 \text { and } \nu^{P^{l-1}}(\gamma, l-1)=\nu_{1}\left(\left.x\right|_{[0,(l-1) T]}\right) \geq 1 .
$$

By Lemma 2.4, $P^{l-1}=I$ and (3.9) -(3.11), we have

$$
\begin{aligned}
i^{I}(\gamma, l-1) & =i^{P^{l-1}}(\gamma, l-1) \\
& \leq i^{P^{l}}(\gamma, l)-i^{P}(\gamma, 1)+\nu(\xi, 1)-\nu(\xi, l)+\frac{e\left(P^{-1} \gamma(T)\right)}{2}+\frac{e\left(P^{-1}\right)}{2}-\nu^{P^{l-1}}(\gamma, l-1) \\
& \leq i^{P^{l}}(\gamma, l)-i^{P}(\gamma, 1)+\frac{e\left(P^{-1} \gamma(T)\right)}{2}+n-1 \\
& \leq i^{P^{l}}(\gamma, l)-i^{P}(\gamma, 1)+2 n-1 .
\end{aligned}
$$

Note that $i^{P^{l}}(\gamma, l)=i^{P}\left(\left.x\right|_{[0, \tau]}\right) \leq \operatorname{dim} \operatorname{ker}_{\mathbb{R}}\left(P-I_{2 n}\right)+1$, here we write $i^{P}\left(\left.x\right|_{[0, \tau]}\right)$ for $i^{P}(x)$ to remind the solution $x$ is defined in the interval $[0, \tau]$. By the definition of Maslov $P$-index,

$$
i^{I}(\gamma, l-1)=i_{1}(\gamma, l-1)+n .
$$


So we get

$$
i_{1}(\gamma, l-1) \leq \operatorname{dim} \operatorname{ker}_{\mathbb{R}}(P-I)-i^{P}(\gamma, 1)+n .
$$

By the condition (H5) and Remark 2.1, we have

$$
i^{P}(\gamma, 1)=i^{P}(B)=\sum_{s \in[0,1)} \nu^{P}(s B)=\sum_{s \in[0,1)} \operatorname{dim} \operatorname{ker}_{\mathbb{R}}\left(\gamma_{B}(s T)-P\right) .
$$

Here we remind that $B(t)$ and $\gamma_{B}$ are defined in (3.8). Since $\gamma_{B}(0)=I$, so $\operatorname{dim} \operatorname{ker}_{\mathbb{R}}\left(\gamma_{B}(s T)-\right.$ $P)=\operatorname{dim} \operatorname{ker}_{\mathbb{R}}(P-I)$ when $s=0$. Thus we have

$$
i^{P}(\gamma, 1) \geq \operatorname{dim} \operatorname{ker}_{\mathbb{R}}(P-I) .
$$

From (3.12), it implies

$$
i_{1}(\gamma, l-1) \leq n
$$

By the convex condition (H5), we also have

$$
i_{1}\left(\left.x\right|_{[0, k T]}\right) \geq n \text { and } i_{1}\left(\left.x\right|_{[0,(l-1) T]}\right) \geq n .
$$

We set $m=\frac{l-1}{k}$. Note that $\left.x\right|_{[0,(l-1) T]}$ is the $m$-th iteration of $\left.x\right|_{[0, k T]}$. By (3.11), (3.16), (3.17) and Lemma 4.1 in [26], we obtain $m=1$ and then $k=l-1$. From the above process (3.12)-(3.13) and (3.15)-(3.17), we obtain $k=l-1$ provided $e\left(P^{-1} \gamma(T)\right)=2 n$, and

$$
\begin{aligned}
i^{P}(\gamma, 1) & =\operatorname{dim}_{\operatorname{ker}_{\mathbb{R}}(P-I) ;} \\
i^{P}(\gamma, l) & =i^{P}(\gamma, k+1)=\operatorname{dim}_{\operatorname{ker}_{\mathbb{R}}}(P-I)+1, \\
\nu^{P^{k}}(\gamma, k) & =\nu^{P}(\gamma, 1)=1 .
\end{aligned}
$$

Here we remind that the left inequality in (2.7) of Lemma 2.3 holds independent of the choice of $\xi \in \mathcal{P}_{\tau}(2 n)$, then for any $\xi \in \mathcal{P}_{\tau}(2 n)$ we have

$$
i^{P}(\gamma, k+1) \geq(k+1)\left(i^{P}(\gamma, 1)+\nu^{P}(\gamma, 1)-n\right)+n-1+(k+1) i_{1}(\xi)-i(\xi, k+1) .
$$

By the condition $P \in S p(2 n)_{k}\left(r, p ; j_{1}, j_{2}, \cdots, j_{r}\right)$, we get

$$
\begin{gathered}
\operatorname{dim} \operatorname{ker}_{\mathbb{R}}(P-I)=2 p, \\
k-2 \sum_{m=1}^{r} m \cdot j_{m}>1 .
\end{gathered}
$$

Applying (3.18), (3.20) and Lemma 3.1 to (3.19), we get

$$
k-2 \sum_{m=1}^{r} m \cdot j_{m} \leq 1 .
$$

It is contradict to the inequality (3.21). So the minimal $P$-symmetric period of $\left(k \tau, x^{k}\right)$ is $k \tau$.

Remark 3.1. Note that $e\left(P^{-1} \gamma(T)\right)=e\left(\left(P^{-1} \gamma(T)\right)^{l}\right)=e\left(P^{-1} \gamma(\tau)\right)=2 n$ is required in the above proof. If $e\left(P^{-1} \gamma(T)\right) \leq 2 n-2$, we get $i_{1}(\gamma, l-1)<n$ by taking the same process as (3.12)-(3.13). It contradicts to the second inequality of 3.17). At this moment, the minimal $P$-symmetric period of $\left(k \tau, x^{k}\right)$ is $k \tau$.

The condition (H5) can be replaced by a weaker condition: $H^{\prime \prime}(x(t)) \geq 0$ and $\int_{0}^{\tau} H^{\prime \prime}(x(t)) d t>$ 0 for the P-solutuin $(\tau, x)$ in Theorem 3.1. 


\section{References}

[1] A.Ambrosetti, V.Coti Zelati, Solutions with minimal period for Hamiltonian systems in a potential well, Ann. Inst. H. Poincaré Anal. Non Linéaire 4 (1987), 242-275.

[2] A.Ambrosetti, G.Mancini, Solutions of minimal period for a class of convex Hamiltonian systems, Math. Ann. 255 (1981), 405-421.

[3] K. C.Chang, Infinite dimensional Morse theory and multiple solution problems, in Progress in Nonlinear Differention Equations and Their Application, Vol.6, (1993).

[4] K. C. Chang,J. Liu and M. Liu, Nontrivial periodic solutions for strong resonance Hamiltonian systems, Ann. Inst. H. Poincaré Anal. Non Linéaire 14(1) (1997), 103-117.

[5] F. Clarke, I. Ekeland, Hamiltonian trajectories having prescribed minimal period, Comm. Pure. Appl. Math. 33 (1980), no.3, 103-116.

[6] A.Chenciner and R.Montgomery, A remarkable periodic solution of the three body problem in the case of equal masses, Ann. of Math. 152: 3 (2000), 881-901.

[7] Y. Dong, P-index theory for linear Hamiltonian systems and multiple solutions for nonlinear Hamiltonian systems, Nonlinearity 19: 6 (2006), 1275-1294.

[8] Y. Dong and Y. Long, Closed characteristics on partially symmetric compact convex hypersurfaces in $R^{2 n}$, J. Diff. Equa. 196 (2004), 226-248.

[9] D. Dong and Y. Long, The iteration formula of the Maslov-type index theory with applications to nonlinear Hamiltonian systems, Trans. Amer. Math. Soc. 349 (1997), 2619-2661.

[10] I. Ekeland, H. Hofer, Periodic solutions with prescribed minimal period for convex autonomous Hamiltonian sysmtems, Invent. Math. 81 (1985), 155-188.

[11] G.Fei, Relative Morse index and its application to Hamiltonian systems in the presence of symmetries, J. Diff. Equa. 122 (1995), 302-315.

[12] G.Fei and Q.Qiu, Minimal period solutions of nonlinear Hamiltonian systems, Nonlinear Analysis, Theory, Method Applications 27: 7 (1996), 821-839.

[13] G.Fei, S. K.Kim, T.Wang Minimal period estimates of period solutions for superquadratic Hamiltonian systmes, J. Math. Anal. Appl. 238 (1999), 216-233.

[14] D. Ferrario and S. Terracini, On the existence of collisionless equivariant minimizers for the classical n-body problem, Invent. Math. 155: 2 (2004), 305-362.

[15] N.Ghoussoub, Location, multiplicity and Morse indices of min-max critical points, J. Reine Angew Math. 417 (1991), 27-76. 
[16] X.Hu and P.Wang, Conditional Fredholm determinant for the S-periodic orbits in Hamiltonian systems, Joural of Functional Analysis 261 (2011), 3247-3278.

[17] X.Hu and S.Sun, Index and stability of symmetric periodic orbits in Hamiltonian systems with application to figure-eight orbit, Comm. Math. Phys. 290 (2009), 737-777.

[18] X. Hu and S. Sun, Morse index and the stability of closed geodesics, Sci. China Math. 53: 5 (2010), 1207-1212.

[19] X.Hu and S.Sun, Stability of relative equilibria and Morse index of central configurations, C. R. Acad. Sci. Paris 347 (2009), 1309-1312.

[20] A.Lazer and S.Solomini, Nontrivial solution of operator equations and Morse indices of critical points of min-max type, Nonlinear Anal. 12 (1988), 761-775.

[21] C.Liu, Maslov P-index theory for a symplectic path with applications, Chin. Ann. Math. 4 (2006), 441-458.

[22] C.Liu, Periodic solutions of asymptotically linear delay differential systems via Hamiltonian systems, J. Diff. Equa. 252 (2012), 5712-5734.

[23] C. Liu, Relative index theories and applications, Top. Meth. Nonl. Anal, to appear.

[24] C. Liu, Minimal period estimates for brake orbits of nonlinear symmetric Hamiltonian systems, Discrete and continuous dynamical systems 27(1) (2010), 337-355.

[25] C.Liu and Y.Long, An optimal increasing estimate of the iterated Maslov-type indices, Chinese Sci. Bull. 42 (1997), 2275-2277.

[26] C.Liu and Y.Long, Iteration inequalities of the Maslov-type index theory with applications, J. Diff. Equa. 165 (2000), 355-376.

[27] C.Liu and S.Tang, Maslov $(P, \omega)$-index theory for symplectic paths, Advanced Nonlinear Studies 15 (2015), 963-990.

[28] C.Liu and S.Tang, Iteration inequalities of the Maslov P-index theory with applications, Nonlinear Analysis 127 (2015), 215-234.

[29] Y.Long, Index theory for symplectic paths with application, Progress in Mathematics, Vol. 207, Birkhäuser Verlag, 2002.

[30] Y.Long, Bott formula of the Maslov-type index theory, Pacific J. Math. 187 (1999), 113-149.

[31] Y. Long, The minimal period problem for classical Hamiltonian systems with even potentials, Ann. Inst. H. Poincaré Anal. non. linéaire 10(1993), 605-626.

[32] Y. Long, The minimal period problem of periodic solutions for autonomous superquadratic second order Hamiltonian systems, J. Diff. Equa. 111(1994), 147-174. 
[33] Y. Long, On the minimal period for periodic solutions of nonlinear Hamiltonian systmes, Chinese Ann. of Math. 18B(1997), 481-484.

[34] Y.Long and C.Zhu, Closed characteristics on compact convex hypersurfaces in $R^{2 n}$, Annals of Math. 155 (2002), 317-368.

[35] P. H.Rabinowitz, Periodic solutions of Hamiltonian systmes, Comm. Pure Appl. Math. 31 (1978), 157-184.

[36] P. H.Rabinowitz, Minimax method in critical point theory with applications to differential equations, CBMS Regional Conf. Ser. in Math., No.65, A. M. S., Providence(1986).

[37] S.Solimini, Morse index estimates in min-max theorems, Manuscription Math. 63 (1989), 421-453. 